\section{Effective Educational Videos: Principles and Guidelines for Maximizing Student Learning from Video Content}

\author{
Cynthia J. Brame* \\ Center for Teaching and Department of Biological Sciences, Vanderbilt University, \\ Nashville, TN 37203
}

\begin{abstract}
Educational videos have become an important part of higher education, providing an important content-delivery tool in many flipped, blended, and online classes. Effective use of video as an educational tool is enhanced when instructors consider three elements: how to manage cognitive load of the video; how to maximize student engagement with the video; and how to promote active learning from the video. This essay reviews literature relevant to each of these principles and suggests practical ways instructors can use these principles when using video as an educational tool.
\end{abstract}

Video has become an important part of higher education. It is integrated as part of traditional courses, serves as a cornerstone of many blended courses, and is often the main information-delivery mechanism in online courses. Several meta-analyses have shown that technology can enhance learning (e.g., Means et al., 2010; Schmid et al., 2014), and multiple studies have shown that video, specifically, can be a highly effective educational tool (e.g., Allen and Smith, 2012; Kay, 2012; Lloyd and Robertson, 2012; Rackaway, 2012; Hsin and Cigas, 2013; Stockwell et al., 2015). Video may have particular value for student preparation in biology classes, in part because students may find it more engaging (Stockwell et al., 2015) and because it can be well suited to illuminating the abstract or hard-to-visualize phenomena that are the focus of so many biology classes (e.g., Dash et al., 2016; see Video Views and Reviews features in CBELife Sciences Education for other examples). The medium is not inherently effective, however; Guo et al. (2014) have shown that students often disregard large segments of educational videos, while MacHardy and Pardos (2015) demonstrate that some videos contribute little to student performance. What, then, are the principles that allow instructors to choose or develop videos that are effective in moving students toward the desired learning outcomes? Consideration of three elements for video design and implementation can help instructors maximize video's utility in the biology classroom:

- Cognitive load

- Student engagement

- Active learning

Together, these elements provide a solid base for the development and use of video as an effective educational tool.

\section{COGNITIVE LOAD}

One of the primary considerations when constructing educational materials, including video, is cognitive load. Cognitive load theory, initially articulated by Sweller (1988, $1989,1994)$, suggests that memory has several components. Sensory memory is transient, collecting information from the environment. Information from sensory memory may be selected for temporary storage and processing in working memory, which has
Kathryn E. Perez, Monitoring Editor Submitted March 15, 2016; Revised May 21, 2016; Accepted May 23, 2016

CBE Life Sci Educ December 1, 2016 15:es6 DOI:10.1187/cbe.16-03-0125

*Address correspondence to: Cynthia J. Brame (cynthia.brameavanderbilt.edu).

() 2016 C. J. Brame. CBE-Life Sciences

Education () 2016 The American Society for Cell Biology. This article is distributed by The American Society for Cell Biology under license from the author(s). It is available to the public under an Attribution-Noncommercial-Share Alike 3.0 Unported Creative Commons License (http:// creativecommons.org/licenses/by-nc-sa/3.0). "ASCB®" and "The American Society for Cell Biology ${ }^{\circledR}$ " are registered trademarks of The American Society for Cell Biology. 
very limited capacity. This processing is a prerequisite for encoding into long-term memory, which has virtually unlimited capacity. Because working memory is very limited, the learner must be selective about what information from sensory memory to pay attention to during the learning process, an observation that has important implications for creating educational materials.

Based on this model of memory, cognitive load theory suggests that any learning experience has three components. The first of these is intrinsic load, which is inherent to the subject under study and is determined in part by the degrees of connectivity within the subject. The common example given to illustrate a subject with low intrinsic load is a word pair (e.g., blue $=a z u l)$; grammar, on the other hand, is a subject with a high intrinsic load due to its many levels of connectivity and conditional relationships. In an example from biology, learning the names of the stages of mitosis would have lower intrinsic load than understanding the process of cell cycle control. The second component of any learning experience is germane load, which is the level of cognitive activity necessary to reach the desired learning outcome-for example, to make the comparisons, do the analysis, and elucidate the steps necessary to master the lesson. The ultimate goal of these activities is for the learner to incorporate the subject under study into a schema of richly connected ideas. The third component of a learning experience is extraneous load, which is cognitive effort that does not help the learner toward the desired learning outcome. It is often characterized as load that arises from a poorly designed lesson (e.g., confusing instructions, extra information) but may also be load that arises due to stereotype threat or imposter syndrome. These concepts are more fully articulated and to some extent critiqued in an excellent review by deJong (2010).

These definitions have implications for design of educational materials and experiences. Specifically, instructors should seek to minimize extraneous cognitive load and should consider the intrinsic cognitive load of the subject when constructing learning experiences, carefully structuring them when the material has high intrinsic load. Because working memory has a limited capacity, and information must be processed by working memory to be encoded in long-term memory, it is important to prompt working memory to accept, process, and send to longterm memory only the most crucial information (Ibrahim et al., 2012).

The cognitive theory of multimedia learning builds on the cognitive load theory, noting that working memory has two channels for information acquisition and processing: a visual/ pictorial channel and an auditory/verbal-processing channel (Mayer, 2001; Mayer and Moreno, 2003). Although each channel has limited capacity, the use of the two channels can facilitate the integration of new information into existing cognitive structures. Using both channels maximizes working memory's capacity-but either channel can be overwhelmed by high cognitive load. Thus, design strategies that manage the cognitive load for both channels in multimedia learning materials promise to enhance learning.

These theories give rise to several recommendations about educational videos (see Table 1). Based on the premise that effective learning experiences minimize extraneous cognitive load, optimize germane cognitive load, and manage intrinsic cognitive lead, four effective practices emerge.
Signaling, which is also known as cueing (deKoning et al., 2009), is the use of on-screen text or symbols to highlight important information. For example, signaling may be provided by the appearance of two or three key words (Mayer and Johnson, 2008; Ibrahim et al., 2012), a change in color or contrast (deKoning et al., 2009), or a symbol that draws attention to a region of a screen (e.g., an arrow; deKoning et al., 2009). By highlighting the key information, signaling helps direct learner attention, thus targeting particular elements of the video for processing in the working memory. This can reduce extraneous load by helping novice learners with the task of determining which elements within a complex tool are important, and it can also increase germane load by emphasizing the organization of and connections within the information. Mayer and Moreno (2003) and deKoning et al. (2009) have shown that this approach improves students' ability to retain and transfer new knowledge from animations, and Ibrahim et al. (2012) have shown that these effects extend to video.

The benefits of signaling are complemented by segmenting, or the chunking of information in a video lesson. Segmenting allows learners to engage with small pieces of new information and gives them control over the flow of new information. As such, it manages intrinsic load and can also increase germane load by emphasizing the structure of the information. Segmenting can be accomplished both by making shorter videos and by including "click forward" pauses within a video, such as using YouTube Annotate or HapYak to provide students with a question and prompting them to click forward after completion. Both types of segmenting have been shown to be important for student engagement with videos (Zhang et al., 2006; Guo et al., 2014) and learning from video (Zhang et al., 2006; Ibrahim et al., 2012).

Weeding, or the elimination of interesting but extraneous information that does not contribute to the learning goal, can provide further benefits. For example, music, complex backgrounds, or extra features within an animation require the learner to judge whether he or she should be paying attention to them, which increases extraneous load and can reduce learning. Importantly, information that increases extraneous load changes as the learner moves from novice toward expert status. That is, information that may be extraneous for a novice learner may actually be helpful for a more expert-like learner, while information that is essential for a novice may serve as an already known distraction for an expert. Thus, it is important that the instructor consider his or her learners when weeding educational videos, including information that is necessary for their processing but eliminating information that they do not need to reach the learning goal and that may overload their working memory. Ibrahim et al. (2012) has shown that this treatment can improve retention and transfer of new information from video.

Finally, the utility of video lessons can be maximized by matching modality to content. By using both the audio/verbal channel and the visual/pictorial channel to convey new information, and by fitting the particular type of information to the most appropriate channel, instructors can enhance the germane cognitive load of a learning experience. For example, showing an animation of a process on screen while narrating it uses both channels to elucidate the process, thus giving the learner dual and complementary streams of information to highlight features 
TABLE 1. Practices to maximize student learning from educational videos

\begin{tabular}{lrr}
\hline $\begin{array}{l}\text { Element to } \\
\text { consider }\end{array}$ & Recommendation & Rationale \\
\hline Cognitive load & $\begin{array}{r}\text { Use signaling to highlight } \\
\text { important information. }\end{array}$ & $\begin{array}{l}\text { Can reduce extraneous load. } \\
\text { Can enhance germane load. }\end{array}$
\end{tabular}
important information.
Use segmenting to chunk information.

Use weeding to eliminate extraneous information.

Match modality by using auditory and visual channels to convey complementary information.

Student Keep each video brief. engagement

Use conversational language.

Speak relatively quickly and with enthusiasm.

Active learning

\section{Consider these strategies for promoting active learning: \\ Packaging video with} interactive questions.

Use interactive features that give students control.

Use guiding questions.

Make video part of a larger homework assignment.
Can enhance germane load.
Manages intrinsic load. Can enhance germane load. Reduces extraneous load.

Can enhance germane load.

Increases percentage of each video that students watch; may increase total watch time.

May decrease mind wandering.

Creates a sense of social partnership between student and instructor, prompting the student to try harder to make sense of the lesson.

Increases percentage of each video that students watch.

May increase sense of social partnership between student and instructor.

Increases percentage of each

May increase germane cognitive load by helping students recognize connections.

May increase germane cognitive load, improve memory via the testing effect, and improve student self-assessment.

Increases student ownership and may increase germane cognitive load.

May increase germane cognitive load, reduce extraneous cognitive load, and improve student self-assessment. video that students watch.

\section{Examples}

Key words on screen highlighting important elements

Changes in color or contrast to emphasize organization of information

Changes in color or contrast to emphasize relationships within information

Brief out-of-video text explaining purpose and context for video (e.g., learning objective for video)

Short videos (6 minutes or less)

Chapters or click-forward questions within videos

Eliminating music

Eliminating complex backgrounds

Khan Academy-style tutorial videos that illustrate and explain phenomena

Narrated animations

Multiple videos for a lesson, each $\leq 6$ minutes

Placing the student in the lesson by use of "your" rather than "the" during explanations

Use of "I" to indicate the narrator's perspective

Speaking rates in the 185-254 words per minute range

Expressions of instructor excitement, such as "I love the next part; the way the feed-forward mechanism works is so elegant," or "Consider how the cell solves this tricky problem of needing to regulate three genes in sequence; it's really cool."

Videos created for the class in which they are going to be used, with instructor narration explaining links to preceding material

Explanatory text to situate video in course

Integrate questions into videos with HapYak or Zaption, as described by Obodo and Baskauf (2015)

Follow short videos with interactive questions within an LMS, as done by Keithly and colleagues (2015), or within Google Forms, as done by Caudel and colleagues (2015)

Create "chapters" within a video using HapYak or YouTube Annotate

Senchina (2011) provides guiding questions for videos designed to introduce physiology students to professional ethics related to experimenter-subject interactions, such as the following: "Observe the subject's behavior and responsiveness during the dehydration period. What changes as the subject becomes dehydrated? What problems does he have? Observe the experimenters' behavior and responsiveness as dehydration progresses. What do they do differently? Why?"

May increase student motivation, Package videos with a series of questions or problems that germane cognitive load, and student self-assessment. ask students to apply the concepts from the videos. iBiology Education videos (e.g., What Can You Learn with a Light Microscope?) provide one example (iBiology, 2016) 
that should be processed in working memory. In contrast, showing the animation while also showing printed text uses only the visual channel and thus overloads this channel and impedes learning (Mayer and Moreno, 2003). In another example, using a "talking head" video to explain a complex process makes productive use only of the verbal channel (because watching the speaker does not convey additional information), whereas a Khan Academy-style tutorial that provides symbolic sketches to illustrate the verbal explanation uses both channels to give complementary information. Using both channels to convey appropriate and complementary information has been shown to increase students' retention and ability to transfer information (Mayer and Moreno, 2003) and to increase student engagement with videos (Guo et al., 2014; Thomson et al., 2014).

\section{STUDENT ENGAGEMENT}

Another lens through which to consider educational video is student engagement. The idea is simple: if students do not watch videos, they cannot learn from them. Lessons on promoting student engagement derive from earlier research on multimedia instruction and more recent work on videos used within MOOCs (massive open online courses; see Table 1).

The first and most important guideline for maximizing student attention to educational video is to keep it short. Guo and colleagues examined the length of time students watched streaming videos within four edX MOOCs, analyzing results from 6.9 million video-watching sessions (Guo et al., 2014). They observed that the median engagement time for videos less than 6 minutes long was close to 100\%-that is, students tended to watch the whole video (although there are significant outliers; see the paper for more complete information). As videos lengthened, however, student engagement dropped, such that the median engagement time with 9- to 12-minute videos was $\sim 50 \%$, and the median engagement time with 12 - to 40 -minute videos was $\sim 20 \%$. In fact, the maximum median engagement time for a video of any length was 6 minutes. Making videos longer than 6-9 minutes is therefore likely to be wasted effort. In complementary work, Risko et al. (2012) showed 1-hour videos to students in a lab setting, probing student self-reports of mind wandering four times in each lecture and testing student retention of lecture material after the lecture. They found that student report of mind wandering increased and retention of material decreased across the video lecture (Risko et al., 2012).

Another method to keep students engaged is to use a conversational style. Called the personalization principle by Mayer, the use of conversational rather than formal language during multimedia instruction has been shown to have a large effect on students' learning, perhaps because a conversational style encourages students to develop a sense of social partnership with the narrator that leads to greater engagement and effort (Mayer, 2008). In addition, some research suggests that it can be important for video narrators to speak relatively quickly and with enthusiasm. In their study examining student engagement with MOOC videos, Guo and colleagues observed that student engagement was dependent on the narrator's speaking rate, with student engagement increasing as speaking rate increased (Guo et al., 2014). It can be tempting for video narrators to speak slowly to help ensure that students grasp important ideas, but including in-video questions, "chapters," and speed control can give students control over this feature-and increasing narrator speed appears to promote student interest.

Instructors can also promote student engagement with educational videos by creating or packaging them in a way that conveys that the material is for these students in this class. One of the benefits for instructors in using educational videos can be the ability to reuse them for other classes and other semesters. When creating or choosing videos, however, it is important to consider whether they were created for the type of environment in which they will be used. For example, a face-to-face classroom session that is videotaped and presented within an online class may feel less engaging than a video that is created with an online environment as the initial target (Guo et al., 2014). A video's adaptability can be enhanced, however: when reusing videos, instructors can package them for a particular class using text outside the video to contextualize the relevance for that particular class and lesson.

\section{ACTIVE LEARNING}

As biology educators, we have abundant evidence that active learning in the classroom provides clear advantages over passive encounters with course material through lecture (e.g., Knight and Wood, 2005; Haak et al., 2011; Freeman et al., 2014). Similarly, elements that promote cognitive activity during video viewing can enhance student learning from this medium (see Table 1).

Schacter and Szpunar (2015) propose a conceptual framework for enhancing learning from educational videos that identifies online learning as a type of self-regulated learning. Self-regulation of learning requires students to monitor their own learning, to identify learning difficulties, and to respond to these judgments; in other words, it requires students to actively build and interrogate mental models, practicing metacognition about the learning process. Novices within a field, however, have difficulty accurately judging their understanding, often overestimating their learning (Bjork et al., 2013). This problem may be enhanced when new information is delivered via video, which students report as easier to learn and more memorable than text (Salomon, 1994; Choi and Johnson, 2005). Incorporating prompts for students to engage in the type of cognitive activity necessary to process information-to engage in active learning - can help them build and test mental models, explicitly converting video watching from a passive to an active-learning event. The means to do this can vary, but the following strategies have demonstrated success in some contexts.

\section{Package Video with Interactive Questions}

Szpunar et al. compared the test performance of students who answered questions interpolated between $\sim 5$ min video lectures and students who did unrelated arithmetic problems between the videos, finding that the students in the interpolated question group performed significantly better on subsequent tests of the material and reported less mind wandering (Szpunar et al., 2013). Students who received the interpolated questions also exhibited increased note taking, reported the learning event as less "mentally taxing," and reported less anxiety about the final test. These results suggest that interpolated questions may improve student learning from video through several mechanisms. First, they may help to optimize cognitive load by decreasing extraneous load (i.e., anxiety about an upcoming 
test) and increasing germane load (i.e., note taking, reduced mind wandering). Further, interpolated questions may produce some of their benefit by tapping into the "testing effect," in which recall of important information strengthens students' memory of and ability to use the recalled information (Roediger and Karpicke, 2006; Brame and Biel, 2015). Finally, interpolated questions may help students engage in more accurate self-assessment (Szpunar et al., 2014), an important benefit for a medium that students may perceive as "easier" than text. Tools like HapYak and Zaption can also allow instructors to embed questions directly into video and to give specific feedback based on student response. This approach has similar benefits to interpolated questions in increasing student performance on subsequent assessments (Vural, 2013) and has the additional benefit of making the video interactive (see following section).

\section{Use Interactive Features That Give Students Control}

Zhang and colleagues compared the impact of interactive and noninteractive video on students' learning in a computer science course (Zhang et al., 2006). Students who were able to control movement through the video, selecting important sections to review and moving backward when desired, demonstrated better achievement of learning outcomes and greater satisfaction. One simple way to achieve this level of interactivity is by using YouTube Annotate, HapYak, or another tool to introduce labeled "chapters" into a video. This not only has the benefit of giving students control but also can demonstrate the organization, increasing the germane load of the lesson.

\section{Use Guiding Questions}

Lawson and colleagues examined the impact of guiding questions on students' learning from a video about social psychology in an introductory psychology class (Lawson et al., 2006). Building on work from Kreiner (1997), they had students in some sections of the course watch the video with no special instructions, while students in other sections of the course were provided with eight guiding questions to consider while watching. The students who answered the guiding questions while watching the video scored significantly higher on a later test. Guiding questions may serve as an implicit means to share learning objectives with students, thus increasing the germane load of the learning task and reducing the extraneous load by focusing student attention on important elements. This strategy is often used to increase student learning from reading assignments (e.g., Tanner, 2012; Round and Campbell, 2013), and it can translate effectively to helping students learn from video.

\section{Make Video Part of a Larger Homework Assignment}

MacHardy and Pardos (2015) have developed a model relating educational video characteristics to students' performance on subsequent assessments. One observation from their analysis of Khan Academy videos was that videos that offered the greatest benefits to students were highly relevant to associated exercises. This result is supported by results observed in a "teaching-as-research" project at Vanderbilt University (for background on teaching as research, see www.cirtl.net). Specifically, Faizan Zubair participated in the BOLD Fellows program, in which graduate students develop online learning materials for incorporation into a faculty mentor's course and then investi- gate their impact in teaching-as-research projects. Zubair developed videos on that were embedded in a larger homework assignment in Paul Laibinis's chemical engineering class and found that students valued the videos and that the videos improved students' understanding of difficult concepts when compared with a semester when the videos were not used in conjunction with the homework (Zubair and Laibinis, 2015; see also Summary).

The important thing to keep in mind is that watching a video can be a passive experience, much as reading can be. To make the most of our educational videos, we need to help students do the processing and self-evaluation that will lead to the learning we want to see.

\section{SUMMARY}

Video may provide a significant means to improve student learning and enhance student engagement in biology courses (Allen and Smith, 2012; Kay, 2012; Lloyd and Robertson, 2012; Rackaway, 2012; Hsin and Cigas, 2013; Stockwell et al., 2015). To maximize the benefit from educational videos, however, it is important to keep in mind the three key components of cognitive load, elements that impact engagement, and elements that promote active learning. Luckily, consideration of these elements converges on a few recommendations:

- Keep videos brief and targeted on learning goals.

- Use audio and visual elements to convey appropriate parts of an explanation; consider how to make these elements complementary rather than redundant.

- Use signaling to highlight important ideas or concepts.

- Use a conversational, enthusiastic style to enhance engagement.

- Embed videos in a context of active learning by using guiding questions, interactive elements, or associated homework assignments.

\section{ACKNOWLEDGMENTS}

This content was largely presented first on the Vanderbilt Center for Teaching website at https://cft.vanderbilt.edu/guides-sub -pages/effective-educational-videos/.

\section{REFERENCES}

Allen WA, Smith AR (2012). Effects of video podcasting on psychomotor and cognitive performance, attitudes and study behavior of student physical therapists. Innov Educ Teach Int 49, 401-414

Bjork RA, Dunlosky J, Kornell N (2013). Self-regulating learning: beliefs, techniques, and illusions. Annu Rev Psychol 64, 417-444.

Brame CJ, Biel R (2015). Test-enhanced learning: the potential for testing to promote greater learning in undergraduate science courses. CBE Life Sci Educ 14, es4

Caudel D, Grundstrom E, Stewart S (2015). AstroNavigation. my.vanderbilt .edu/astronav/overview/coordinates (accessed 16 May 2016)

Choi HJ, Johnson SD (2005). The effect of context-based video instruction on learning and motivation in online courses. Am J Dist Educ 19, 215227.

Dash S, Kamath U, Rao G, Prakash J, Mishra S (2016). Audio-visual aid in teaching "fatty liver." Biochem Mol Biol Educ 44, 241-245.

deJong $T$ (2010). Cognitive load theory, educational research, and instructional design: some food for thought. Instr Sci 38, 105-134. 
deKoning B, Tabbers H, Rikers R, Paas F (2009). Towards a framework for attention cueing in instructional animations: guidelines for research and design. Educ Psychol Rev 21, 113-140.

Freeman S, Eddy SL, McDonough M, Smith MK, Okoroafor N, Jordt H, Wenderoth MP (2014). Active learning increases student performance in science, engineering, and mathematics. Proc Natl Acad Sci USA 111, 8410-8415.

Guo PJ, Kim J, Robin R (2014). How video production affects student engagement: an empirical study of MOOC videos. L@S'14 Proceedings of the First ACM Conference on Learning at Scale, New York: ACM, 41-50.

Haak DC, HilleRisLambers J, Pitre E, Freeman S (2011). Increased structure and active learning reduce the achievement gap in introductory biology. Science 332, 1213-1216.

Hsin WJ, Cigas J (2013). Short videos improve student learning in online education. J Comput Sci Coll 28, 253-259.

iBiology (2016). iBioEducation: iBiology Courses. www.ibiology.org/ ibioeducation.html (accessed 16 May 2016).

Ibrahim M, Antonenko PD, Greenwood CM, Wheeler D (2012). Effects of segmenting, signaling, and weeding on learning from educational video. Learn Media Technol 37, 220-235.

Kay RH (2012). Exploring the use of video podcasts in education: a comprehensive review of the literature. Comput Human Behav 28, 820-831.

Keithly M, Sebastian T, Woelfle M, Friedman K (2015). Blending it up: active learning in a STEM classroom through the use of online materials, BOLD Fellows Project Gallery. https://vanderbilt.edu/bold/docs/bsci-2210 -principles-of-genetics (accessed 15 October 2016).

Knight JK, Wood WB (2005). Teaching more by lecturing less. Cell Biol Educ 4, 298-310

Kreiner DS (1997). Guided notes and interactive methods for teaching with videotapes. Teach Psychol 24, 183-185

Lawson TJ, Bodle JH, Houlette MA, Haubner RR (2006). Guiding questions enhance student learning from educational videos. Teach Psychol 33, $31-33$.

Lloyd SA, Robertson CL (2012). Screencast tutorials enhance student learning of statistics. Teach Psychol 39, 67-71.

MacHardy Z, Pardos ZA (2015). Evaluating the relevance of educational videos using BKT and big data. In: Proceedings of the 8th International Conference on Educational Data Mining, Madrid, Spain, ed. OC Santos, JG Boticario, C Romero, M Pechenizkiy, A Merceron, P Mitros, JM Luna, C Mihaescu, P Moreno, A Hershkovitz, S Ventura, and M Desmarais, http:// educationaldatamining.org/EDM2015/index.php?page=proceedings (accessed 15 October 2016).

Mayer RE (2001). Multimedia Learning, New York: Cambridge University Press.

Mayer RE (2008). Applying the science of learning: evidence-based principles for the design of multimedia instruction. Cogn Instr 19, 177-213.

Mayer RE, Johnson Cl (2008). Revising the redundancy principle in multimedia learning. Br J Educ Psychol 100, 380-386.

Mayer RE, Moreno R (2003). Nine ways to reduce cognitive load in multimedia learning. Educ Psychol 38, 43-52.

Means B, Toyama Y, Murphy R, Bakia M, Jones K (2010). Evaluation of Evidence-Based Practices in Online Learning: Meta-Analysis and Review of Online Learning Studies, Washington, DC: US Department of Education.
Obodo U, Baskauf S (2015). BSCI 111b: Introduction to Biological Sciences Laboratory, BOLD Fellows Project Gallery. https://vanderbilt.edu/bold/ docs/bsci-111b-introduction-to-biological-sciences-laboratory (accessed 16 May 2016)

Rackaway C (2012). Video killed the textbook star? Use of multimedia supplements to enhance student learning. J Pol Sci Educ 8, 189-200.

Risko EF, Anderson N, Sarwal A, Engelhardt M, Kingstone A (2012). Everyday attention: variation in mind wandering and memory in a lecture. Appl Cognitive Psych 26, 234-242.

Roediger HL, Karpicke JD (2006). The power of testing memory: basic research and implications for educational practice. Perspect Psychol Sci 1 , 181-210.

Round JE, Campbell AM (2013). Figure facts: encouraging undergraduates to take a data-centered approach to reading primary literature. CBE Life SCi Educ 12, 39-46

Salomon G (1994). Interaction of media, cognition, and learning, Hillsdale, NJ: Lawrence Erlbaum Associates.

Schacter DL, Szpunar KK (2015). Enhancing attention and memory during video-recorded lectures. Sch Teach Learn Psychol 1, 60-71.

Schmid RF, Bernard RM, Borokhovski E, Tamim RM, Abrami PC, Surkes MA Wade CA, Woods J (2014). The effects of technology use in postsecondary education: a meta-analysis of classroom applications. Comput Educ 72, 271-291.

Senchina DS (2011). Video laboratories for the teaching and learning of professional ethics in exercise physiology curricula. Adv Physiol Educ 35, 264-269.

Stockwell BR, Stockwell MS, Cennamo M, Jiang E (2015). Blended learning improves science education. Cell 162, 933-936.

Sweller J (1988). Cognitive load during problem solving: effects on learning Cogn Sci 12, 257-285

Sweller J (1989). Cognitive technology: some procedures for facilitating learning and problem-solving in mathematics and science. $\mathrm{Br} \mathrm{J}$ Educ Psychol 81, 457-466.

Sweller J (1994). Cognitive load theory, learning difficulty, and instructional design. Learn Instr 4, 295-312.

Szpunar KK, Jing HG, Schacter DL (2014). Overcoming overconfidence in learning from video-recorded lectures: implications of interpolated testing for online education. J Appl Res Mem Cogn 3, 161-164.

Szpunar KK, Khan NY, Schacter DL (2013). Interpolated memory tests reduce mind wandering and improve learning of online lectures. Proc Natl Acad Sci USA 110, 6313-6317.

Tanner KD (2012). Promoting student metacognition. CBE Life Sci Educ 11, 113-120.

Thomson A, Bridgstock R, Willems C (2014). "Teachers flipping out" beyond the online lecture: maximising the educational potential of video. J Learn Des 7, 67-78.

Vural OF (2013). The impact of a question-embedded video-based learning tool on e-learning. Educ Sci Theory Pract 13, 1315-1323.

Zhang D, Zhou L, Briggs RO, Nunamaker JF Jr (2006). Instructional video in e-learning: assessing the impact of interactive video on learning effectiveness. Inf Manage 43, 15-27.

Zubair F, Laibinis P (2015). Interactive videos to enhance student understanding of thermodynamic efficiency. Presentation at the American Institute of Chemical Engineers, held 11 November 2015 in Salt Lake City, UT. 